\title{
Evidence for ferromagnetic order at gadolinium surfaces above the bulk Curie temperature
}

\author{
C. Rau \\ Physics Department, Rice University, Houston, Texas 77251 \\ S. Eichner \\ Sektion Physik, University Munich, Munich, Federal Republic of Germany
}

(Received 9 June 1986)

\begin{abstract}
The electron-spin polarization $P$ at the topmost layer of atomically clean, magnetized gadolinium (Gd) surfaces is measured by using electron capture spectroscopy. $P$ varies almost linearly with temperature between $160 \mathrm{~K}(P=-42 \%)$ and $315 \mathrm{~K}(P=0)$. It is found that the surface Curie temperature $T_{C s}$ is far above-lying near $310 \mathrm{~K}$-the bulk Curie temperature $T_{C b}=292.5 \mathrm{~K}$, indicating uniaxial anisotropic magnetic exchange interactions at the topmost surface layer of $\mathrm{Gd}$.
\end{abstract}

There is presently considerable interest in ordering phenomena in two dimensions. Such ordering can occur in magnets, in fluids, and in solids. ${ }^{1-3}$

For magnetic systems, the influence of a twodimensional surface on phase transitions has been investigated theoretically by numerous authors using various models. ${ }^{1-3}$ However, there is a severe lack of experimental information, in particular concerning the temperature dependence of surface magnetic order. This is primarily due to the enormous difficulties in preparing atomically clean surfaces which remain uncontaminated and well defined during the multiple heating and cooling cycles that are prerequisites for reliable data.

In this paper we report electron-spin polarization (ESP) measurements performed at the topmost layer of atomically clean surfaces of the rare-earth metal Gd which is the prototype of an isotropic Heisenberg ferromagnet. The important question we address is this: Does isotropic magnetic behavior change in going from bulk to surface?

We demonstrate in this work that, for Gd, the topmost surface layer is magnetically ordered while the bulk is disordered. We use electron capture spectroscopy ${ }^{4,5}$ (ECS) and show that at $\mathbf{G d}$ surfaces long-range ferromagnetic order exists far above the bulk Curie temperature $T_{C b}$.

The basic process in ECS is the capture of spinpolarized electrons during grazing-angle reflection of fast (150-keV) deuterons. The minimum distance of the ions towards the reflecting surface amounts to about $0.2 \mathrm{~nm}$ (incidence angle of $0.2^{\circ}$ ), showing that in real-space the ions probe only the exponential tail of the electronic wave functions at the surface. This reveals the extreme surface sensitivity of ECS. Further details on this technique are given in Ref. 5.

For the detection of long-range surface magnetic order, we make use of one-electron capture processes: Surface electrons are captured by fast deuterons at well-defined surfaces of ferromagnetics magnetized parallel to the surface plane. Defining the electron-spin polarization $P$ parallel to the direction of the internal magnetizing field yields

$$
P=\left(n^{+}-n^{-}\right) /\left(n^{+}+n^{-}\right),
$$

where $n^{+}$and $n^{-}$denote the electron numbers with magnetic moment parallel and antiparallel, respectively, to the magnetizing field, which is also used as quantization axis.

The ESP measurements are performed at atomically clean surfaces of 5000 - $\AA$-thick Gd films evaporated in situ on various substrates (nonmagnetic $\mathrm{Cu}$ and magnetic $\mathrm{Gd})$. The $\mathrm{C}$ and $\mathrm{O}$ contamination of the films is less than 0.01 monolayer, as monitored with Auger electron spectroscopy after the film preparation and also between the measuring cycles. $T_{C b}$ is measured in situ by an inductive method. We find $T_{C b}=292.5 \mathrm{~K}$. At each selected value of the temperature $T$, the temperature of a specimen is kept constant within $0.01 \mathrm{~K}$ by an automatic regulation device which is absolutely calibrated within $0.1 \mathrm{~K}$. Kerr effect measurements show a single Weiss domain at the surface of the specimen for all applied internal magnetic fields ( $\geq 24 \mathrm{kA} / \mathrm{m}=300 \mathrm{Oe}$ ).

The magnetization of a sample is achieved by an internal magnetic field $H_{i}^{\mathrm{Gd}}$ using a closed magnetic circuit consisting of an iron toroid and of the specimen. For the evaluation of $H_{i}^{\mathrm{Gd}}$, we calculate the total magnetic flux $\phi$ in the circuit which is generated by a magnetizing coil placed coaxially around the iron toroid. $\phi$ is given by $\phi=\mu_{0} N I / \sum_{x} R_{x}^{m}$, with $N$ the number of coil windings, $I$ the current in the coil, and $R_{x}^{m}=l_{x} /\left(\mu_{x} A_{x}\right)$ the magnetic resistivity of an element $x$ (iron toroid, $x=\mathrm{Fe}$; specimen, $x=\mathrm{Gd}$ ) in the circuit ( $l_{x}$ and $A_{x}$ are the length and area of $x$ parallel and normal, respectively, to the flux density lines; $\mu_{x}$ is the permeability of $x$ ). $\phi$ is also equal to

$$
\phi=\mu_{0} \mu_{\mathrm{Gd}} H_{i}^{\mathrm{Gd}} A_{\mathrm{Gd}}=\mu_{0} \mu_{\mathrm{Fe}} H_{i}^{\mathrm{Fe}} A_{\mathrm{Fe}},
$$

where $\mu_{\mathrm{Gd}}$ and $\mu_{\mathrm{Fe}}$ depend both on $T$ and on $H_{i}^{\mathrm{Gd}}$, respectively, $H_{i}^{\text {Fe }}$ being the internal magnetic field in the iron toroid. Using numerical values for $\mu_{\mathrm{Gd}}\left(T, H_{i}^{\mathrm{gd}}\right)$ evaluated from magnetization measurements, ${ }^{6,7}$ and taking tabulated data for $\mu_{\mathrm{Fe}}\left(T, H_{i}^{\mathrm{Fe}}\right)$ from Ref. 8, enables us to determine $H_{i}^{\mathrm{Gd}}(I)$. In the following, the symbol $H$ is used for $H_{i}^{\mathrm{Gd}}$.

Figure 1(b) shows the electron-spin polarization $P(T, H)$ measured with $H=48 \mathrm{kA} / \mathrm{m}(600 \mathrm{Oe})$, using atomically clean surfaces of $\mathbf{G d}$ films evaporated on $\mathrm{Gd}$ 


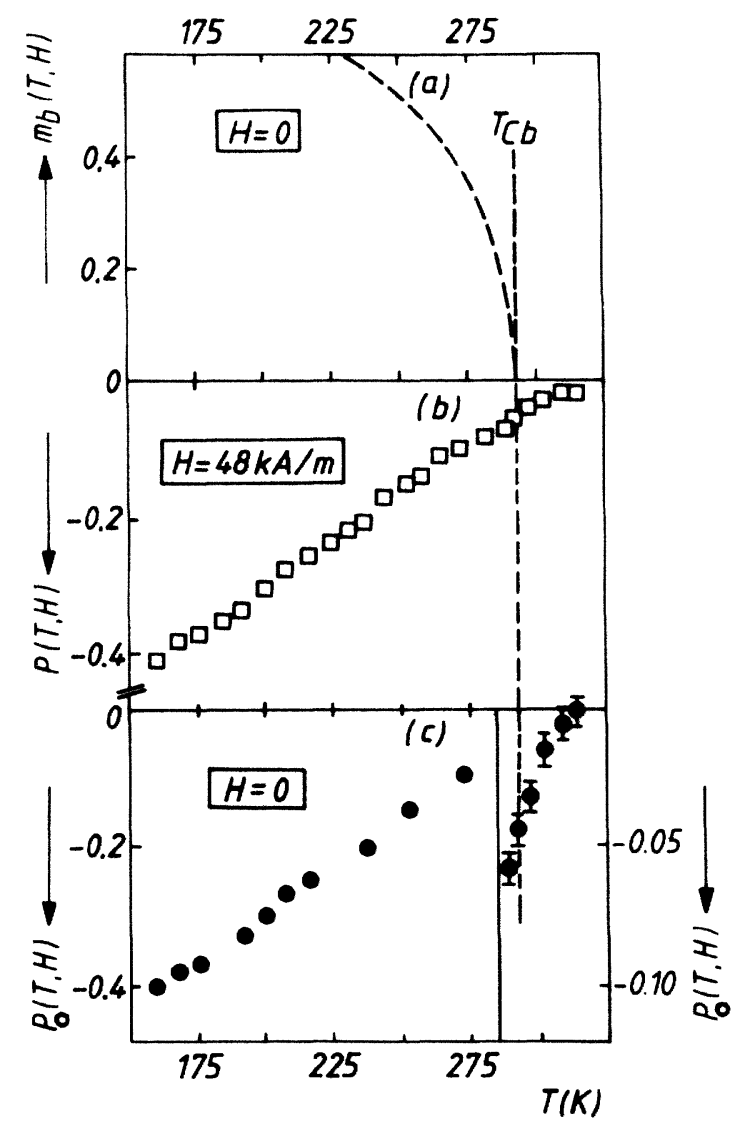

FIG. 1. (a) Temperature dependence of the bulk spontaneous magnetization $m_{b}(T, H=0)$ for Gd, taken from Fig. 6 of Ref. 6; (b) temperature dependence of the electron-spin polarization $P(T, H)$ for $H=48 \mathrm{kA} / \mathrm{m}$; (c) temperature dependence of the spontaneous electron-spin polarization $P(T)$ using $T_{C s}=315 \mathrm{~K}$ for the extrapolation.

substrates. A preliminary account of some results has appeared in Ref. 9(a). Note that an oxidized Gd surface exhibits zero polarization.

These data are obtained from numerous heating and cooling cycles. At each temperature, the polarization data are reproducible within the experimental errors $( \pm 0.45 \%)$, which are only due to counting statistics. In order to make sure whether there is any flux focusing effect in the $\mathrm{Gd}$ films, we used also nonmagnetic $\mathrm{Cu}$ as a substrate. In that case, the polarization values exhibit no significant differences compared to those obtained at the surface of Gd films evaporated on Gd. This clearly indicates that magnetic flux focusing effects in the films play no role in the experiment, contrary to what the high $P$ values above $T_{C b}$ may suggest.

At this point, we note two important features of these measurements:

(1) The existence of a nonzero polarization below $T_{C b}$ is in agreement with Ruderman-Kittel-Kasuya-Yosida (RKKY) (Ref. 10) theory, in which a spin polarization of the $(5 d, 6 s)$ conduction electrons is predicted. The negative sign of the ESP measured at a polycrystalline surface is a very interesting fact, implying directly that the polarization at single-crystalline Gd surfaces should give a negative ESP at least for one low-index surface plane. ${ }^{9(b)}$

(2) The temperature dependence of $P$ at the topmost surface layer [see Fig. 1(b)] is drastically different from the bulk magnetization [see Fig. 1(a)]. Between 160 and approximately $300 \mathrm{~K}, P$ changes almost linearly with temperature $T$. We note that neutron-diffraction experiments ${ }^{11}$ show that below and above $T_{C b}$, the magnetization of the $4 f$ electrons in the bulk is proportional to the total conduction electron polarization, which is again proportional to the polarization of the Fermi electrons. This should also hold for the surface region. ${ }^{12}$ Therefore the measured $P$ values should reveal the temperature behavior of the magnetization of the $4 f$ spins in the topmost surface layer. The proportionality between the $4 f$ magnetization and the polarization of the Fermi electrons implies that the exchange splitting between the spin-up and spindown electron bands is temperature independent, as predicted by "local" band theory ${ }^{13}$ and recently found in electron-capture spectroscopy and photoelectron emission experiments. ${ }^{4,14}$

For the analysis of the experimental data, we first assume that the Gd surface orders with the bulk $\left(T_{C s}=T_{C b}\right)$. In this case, the measured nonzero polarization values for $H \neq 0$ above $T_{C b}$ should be correlated to the tail of the magnetization $m_{b}(T, H)$ of the sample, which always exists above $T_{C b}$ for $H \neq 0$. For the calculation of the normalized $\operatorname{ESP} p(T, H)=P(T, H) / P^{\text {sat }},{ }^{15}$ we use $P^{\text {sat }}=-87 \%$, which is derived from a linear extrapolation of the $P$ values towards $T \rightarrow 0$. Note that if $P^{\text {sat }}$ is smaller than $-87 \%$, then the calculated $p(T, H)$ data, where $P^{\text {sat }}=-87 \%$ is used, should be interpreted as the lowest values for $p(T, H)$. In Table I we have listed, for the interesting temperature region between 292.5 and 315 $\mathrm{K}$, values of $p(T, H)$ for $H=24 \mathrm{kA} / \mathrm{m}(=300 \mathrm{Oe})$, together with data for the normalized bulk magnetization $m_{b}(T, H)=M_{b}(T, H) / M_{b}^{\text {sat }},{ }^{15}$ where $M_{b}(T, H)$ is the bulk magnetization.

The value of

$$
m_{b}(T, H=24 \mathrm{kA} / \mathrm{m})=M_{b}(T, H=24 \mathrm{kA} / \mathrm{m}) / M_{b}^{\text {sat }}
$$

TABLE I. Comparison of $m_{b}(T, H)$ and $p(T, H)$ data for an applied internal magnetizing filed $H=24 \mathrm{kA} / \mathrm{m}$.

\begin{tabular}{lrrrrl}
\hline \hline$T(\mathbf{K})$ & $292.5^{\mathrm{a}}$ & 298 & 303 & 310 & 315 \\
$m_{b}(T, H)^{\mathrm{b}}$ & 0.090 & 0.020 & 0.010 & 0.006 & \\
$m_{b}(T, H)^{\mathrm{c}}$ & 0.031 & 0.005 & 0.001 & 0 & 0 \\
$p(T, H)$ & 0.055 & 0.041 & 0.024 & 0.010 & 0.007 \\
\hline \hline
\end{tabular}

${ }^{\mathrm{a}} T_{\mathrm{Cb}}=292.5 \mathrm{~K}$.

${ }^{b}$ Evaluated from Ref. 7.

${ }^{\mathrm{c}}$ Evaluated from Ref. 6. 


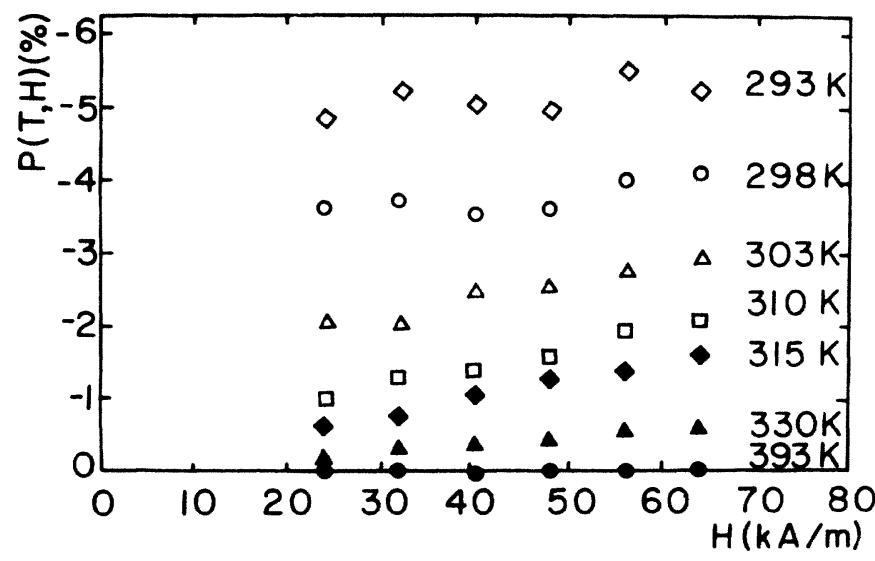

FIG. 2. Electron-spin polarization isotherms $P(T, H)$ as a function of the internal magnetizing field $H$.

is taken from experimental values of the bulk magnetization $M_{b}(T, H)$ at $H=24 \mathrm{kA} / \mathrm{m} .^{6,7}$ From Table I we see that for $T=303 \mathrm{~K}$, e.g., $p$ is at least 2.4 times larger than $m_{b}$, which clearly shows that the measured ESP values above $T_{C b}$ cannot be linked to the tail of $m_{b}$ for $H \neq 0$. Consequently, the measured $P$ values above $T_{C b}$ must be attributed to the existence of ferromagnetic order above $T_{C b}\left(T_{C s}>T_{C b}\right)$.

Further evidence for this conclusion can be obtained by evaluating data for the spontaneous polarization $P_{0}(T)=P(T, H=0)$. To further test the above interpretation of these data, we have performed more detailed $P(T, H)$ measurements in the critical temperature region (see Fig. 2).

In terms of the reduced temperature $t=\left|T_{\mathrm{cr}}-T\right| / T_{\mathrm{cr}}$ and the internal normalized magnetic field $h=\mu_{0} \mu_{a} H$ / $k T_{\mathrm{cr}} \quad\left(\mu_{a}\right.$ is the atomic magnetic moment, $\left.k=1.38 \times 10^{-23} \mathrm{~J} / \mathrm{K}\right), M(t, h)$ is, from scaling theory, ${ }^{16} \mathrm{a}$ linear function of $h$ in the region $t \gg h .^{17}$ This linear re- lation is used to determine $M_{0}(T)$ by extrapolation towards $h \rightarrow 0$ of the $M(T, H)$ data for a single Weissdomain specimen.

Assuming that $T_{C s}=T_{C b}$, the condition $t \gg h$ $\left[H \ll\left(k / \mu_{0} \mu_{a}\right)\left|T_{\mathrm{cr}}-T\right|\right]$ is satisfied for $T \geq 298 \mathrm{~K}$. From the $P(H)$ values at 298,303 , and $310 \mathrm{~K}$ (see Fig. 2), it is clear that the $P(H)$ values do not extrapolate to zero, establishing unambiguously $T_{C s} \neq T_{C b}$.

Likewise, assuming $T_{C s}=298 \mathrm{~K}$ would lead to a vanishing polarization $P(T)$ as $H \rightarrow 0$ for $T=303 \mathrm{~K}$, which is clearly disproved by the data. From these considerations and the data of Fig. 2, we conclude that $T_{C s}$ lies between 303 and $315 \mathrm{~K}$.

The spontaneous polarization $P_{0}(T)$ is shown in Fig. 1(c) for $T_{C s}=315 \mathrm{~K}$. For comparison, we show in Fig. 1(a) the temperature dependence of the reduced bulk spontaneous magnetization $m_{b 0}(T)=m_{b}(T, H=0)$, taken from Fig. 6 of Ref. $7\left[m_{b 0}(T=0)=1\right]$.

It may be mentioned that, in a recent theoretical work, Selzer and Majlis ${ }^{18}$ studied the effect of anisotropy in the surface exchange interaction of a Heisenberg ferromagnet with energy $J\left(S_{i}^{x} S_{j}^{x}+S_{i}^{y} S_{j}^{y}+\eta S_{i}^{z} S_{j}^{z}\right)$, where $J$ is the surface exchange-coupling constant and $\eta$ measures the anisotropy of the exchange interaction in the topmost surface layer. These authors find that for $\eta$ larger than a critical value of $1.41, T_{C s}$ is above $T_{C b}$.

Our results for $\mathrm{Gd}$ are consistent with $\eta$ ranging between 1.51 and 1.58. The shape of the $P(T, H)$ and $P_{0}(T)$ curves [see Figs. 1(b) and 1(c)] for temperatures near $T_{C b}$ reflects the onset of bulk magnetization as evaluated by Selzer and Majlis. A related experiment has been performed $^{19}$ in which, using spin-polarized low-energy electron diffraction at Gd surfaces, surface Curie temperatures between 305 and $315 \mathrm{~K}$ have been observed.

One of us (C.R.) is indebted to J. S. Helman and M. Robert for numerous very stimulating and clarifying discussions and is grateful to H. C. Siegmann for his constructive criticism.
${ }^{1}$ K. Binder and P. Hohenberg, Phys. Rev. B 9, 2194 (1974); 6, 3461 (1972); and D. L. Mills, ibid. 3, 3887 (1971).

${ }^{2}$ T. W. Burkhardt and E. Eisenriegler, Phys. Rev. B 16, 3213 (1977).

${ }^{3}$ M. E. Fisher, in Proceedings of the 1982 Sanibel Symposia, Part III, edited by P. O. Lowdin (Wiley, New York, 1982), [Int. J. Quantum Chem., Symp. 16, 671 (1982)]; H. Nakanishi and M. E. Fisher, Phys. Rev. Lett. 49, 1565 (1982); and references cited in Refs. 1-3.

${ }^{4}$ C. Rau and S. Eichner, Phys. Rev. Lett. 47, 939 (1981).

${ }^{5}$ C. Rau, J. Magn. Magn. Mater. 30, 141 (1982).

${ }^{6}$ H. E. Nigh, S. Legvold, and F. H. Spedding, Phys. Rev. 132, 1092 (1963).

${ }^{7}$ C. D. Graham, Jr., J. Appl. Phys. 36, 1135 (1965).

${ }^{8}$ L. Bergmann and C. Schäfer, Lehrbuch der Experimentalphysik (de Gruyter, Berlin, 1966), Vol. 2.

9(a) C. Rau and S. Eichner, Nuclear Methods in Materials Research, edited by K. Bethge, H. Baumann, H. Jex, and F.
Rauch (Vieweg, Braunschweig, 1980), p. 354. (b) Using polycrystalline surfaces, significant $(h k l)$-dependent information may be lost. The measurement then yields an average value for the ESP which makes the comparison with theoretical calculations of the ESP and also the comparison of ESP measurements with other ESP-sensitive methods difficult.

${ }^{10}$ A. J. Freeman, in Magnetic Properties of Rare Earth Metals, edited by R. J. Elliot (Plenum, New York, 1972).

${ }^{11}$ R. M. Moon, W. C. Koehler, J. W. Cable, and H. R. Child, Phys. Rev. B 5, 997 (1972).

12R. L. Kautz and B. B. Schwartz, Phys. Rev. B 14, 2017 (1976).

${ }^{13}$ V. Korenman, J. L. Murray, and R. E. Prange, Phys. Rev. B 16, 4032 (1977); 16, 4048 (1977); 16, 4058 (1977).

${ }^{14}$ D. E. Eastman, F. J. Himpsel, and J. A. Knapp, Phys. Rev. Lett. 40, 1514 (1978).

${ }^{15} p^{\text {sat }}$ and $M_{b}^{\text {sat }}$ are the saturation values of $P(T, H)$ and $M(T, H)$ at $T=0$. 
16B. Widom, J. Chem. Phys. 43, 3898 (1965).

17J. Balberg and J. S. Helman, Phys. Rev. B 18, 303 (1978); see Table I.

${ }^{18}$ S. Selzer and N. Majlis, Phys. Rev. B 27, 554 (1983). It is of interest to note that the coexistence of an ordered surface phase with a disordered bulk phase can be established very simply using correlation inequalities for the case of the Ising and the $X Y$ models: See D. B. Abraham and C. E. Pfister, Phys. Lett. 96A, 243 (1983).

${ }^{19}$ D. Weller, S. F. Alvarado, W. Gudat, K. Schröder, and M. Campagna, Phys. Rev. Lett. 54, 1555 (1985). 